\title{
Free Vibration Analysis of Elastic Bars using Isogeometric Approach
}

\section{Sang Jin LEE and Kyoung-Sub Park}

ADOPT Research Group, Department of Architectural Engineering, Gyeongsang National University

\begin{abstract}
A study on the free vibration analysis of elastic bar is described in this paper. In order to determine the natural frequencies of bars, a bar element is developed by using isogeometric formulation. The B-spline is introduced to represent the geometry of bar and the same geometric definition is also used to define its unknown displacement field in isogeometric formulation. Therefore, the stiffness and mass matrices are derived by the order-free B-spline basis function. The efficiency and accuracy of the present isogeometric bar elementis demonstrated by using several numerical tests. From numerical results, it is found to be that the present isogeometric element produces very accurate natural frequencies of bars. Finally, the present isogeometric solutions are provided as future reference solutions.
\end{abstract}

Keywords: Isogeometric Analysis, Elastic Bar, Free Vibration, NURBS, Knot Vector, Control Point

\section{INTRODUCTION}

The isogeometric analysis technique (Hughes et al, 2005) has been recently introduced in engineering and science disciplines and many researchers working in numerical analysis field are paying much attention on it. It has been gradually adopted in structural engineering and now being considered as a novel analysis technique for engineering structures. In isogeometric formulation, the B-spline (De Boor, 1978) has been used to model the structural geometry and also to represent the deformed shape of the structures.

The B-spline can provide higher continuity of derivatives than Lagrange interpolation functions that is broadly used in finite element

\section{Corresponding Author: Sang Jin LEE, Professor \\ Department of Architectural Engineering, Gyeongsang \\ National University \\ Gajwa-dong, Jinju-si, Gyeongsangnam-do, 660-701, Korea \\ Tel :+82 557516541 e-mail: lee@gnu.ac.kr}

This research was supported by a grant (code\#06 R\&D B03: 09CHUD-B043631-04-000000) from Cutting-edge Urban Development Program funded by the Ministry of Land, Transport and Maritime Affairs of Korean government. The first author is also gratefully acknowledged for research grant from Gyeongsang National University for his sabbatical leave to University of Cambridge

This is an Open Access article distributed under the terms of the Creative Commons Attribution Non-Commercial License (http://creativecommons. org/licenses/by-nc/3.0/) which permits unrestricted non-commercial use, distribution, and reproduction in any medium, provided the original work is properly cited.
(FE) formulation. In addition, the B-spline basis function can be refined and its order can be easily elevated without changing the geometry or its parameterization (Rogers, 2000). Therefore, the $\mathrm{h}$ - and p-refinement schemes can be used in efficient way and the so-called k-refinement can be also utilized in the analysis (Cottrell et al, 2009).

The applications of isogeometric concept to specific structural analysis problem is few in open literatures since most of recent works have focused on the demonstration of the capability of this new concept for general cases (Hughes and Evans, 2010). Therefore, the application of the isogeometric concept to the analysis of structural members such as bar and beam is still demanding task and the detailed isogeometric solutions for bar and beam is necessarily required.

In this context, we introduce isogeometric approach to evaluate the free vibration behavior of elastic bars. For this purpose, isogeometric bar element is developed here and the isogeometric formulation of elastic bar under free vibration and its implementation procedure are provided here. The present isogeometric element is then tested with several numerical examples. The convergence rate of the present isogeometric bar element is demonstrated by using different refinement strategies such as h-, p- and k-refinements and the frequency spectra are evaluated with the different orders of basis function. Finally, the present isogeometric solutions are provided as future reference solution.

\section{B-SPLINES}

\subsection{Knot vector}

A knot vector $\Xi$ is a set of non-decreasing real values that constitutes a set of coordinates in the parametric space: 


$$
\Xi=\left[\xi_{1}, \xi_{2}, \xi_{3}, \ldots, \xi_{n+p+1}\right]
$$

where $\mathrm{n}$ is the number of basis functions and $\mathrm{p}$ is the order of the B-spline. A knot vector is said to be uniform if its knots are uniformly spaced and non-uniform otherwise. Moreover, a knot vector is said to be open if its first and last knots are repeated $p+1$ times. Basis functions formed from open knot vectors are interpolatory at the ends of the parametric interval $\left[\xi_{1}, \xi_{n+p+1}\right]$ but are not, in general, interpolatory at interior knots. It should be noted that we would employ open knot vectors throughout the analysis of elastic bar.

\subsection{Basis functions}

$\mathrm{B}$-spline basis function is defined recursively starting with $\mathrm{p}=0$ as:

For $\mathrm{p} \geq 1$,

$$
N_{i, 0}(\xi)=\left\{\begin{array}{lr}
1 & \text { if } \xi_{i} \leq \xi<\xi_{i+1} \\
0 & \text { otherwise }
\end{array}\right.
$$

$$
\begin{aligned}
N_{i, p}(\xi)= & \frac{\xi-\xi_{i}}{\xi_{i+p}-\xi_{i}} N_{i, p-1}(\xi) \\
& +\frac{\xi_{i+p+1}-\xi}{\xi_{i+p+1}-\xi_{i+1}} N_{i+1, p-1}(\xi)
\end{aligned}
$$

As an example, we illustrate the quadratic basis functions having control points $\mathrm{n}=8$ generated from the open knot vector $\Xi=[0,0,0,0$ .2,0.4,0.6,0.8,0.8,1,1,1] (see Figure 1).

It is well-known that they are $\mathrm{C}^{\mathrm{p}-1}$-continuous if internal knots are not repeated. However, if a knot has multiplicity $\mathrm{k}$, the function is $\mathrm{C}^{\mathrm{p}-}$ ${ }^{k}$-continuous at the particular knot. For example, when a knot has multiplicity $\mathrm{p}$, the basis function is $\mathrm{C}^{0}$ and interpolatory at that location.

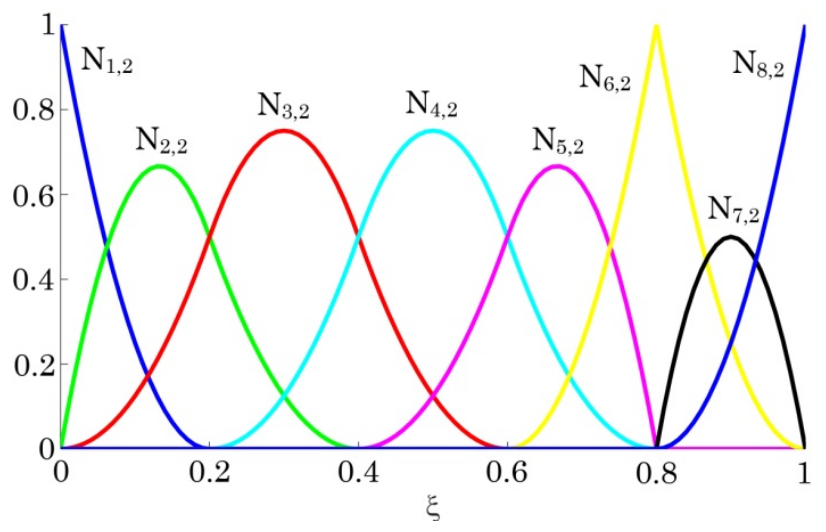

Figure 1. Basis function for B-spline curve

\subsection{B-spline curves}

We construct $\mathrm{n}$ basis functions with the order of a B-spline and an appropriately defined knot vector, The piecewise polynomial B-spline curve $S(\xi)$ of order $p$ can be obtained by taking a linear combination of basis function and control points:

$$
S(\xi)=\sum_{i=1}^{n} N_{i, p}(\xi) C_{i}
$$

where $\mathrm{C}^{\mathrm{i}}$ is the $\mathrm{i}^{\text {th }}$ control point. The piecewise linear interpolation of the control points defines the control net.

As shown in Figure 2, a quadratic 2-dimensional B-spline curve generated from the basis functions shown in Figure 1 along with its control net. A B-spline curve has continuous derivatives of order $\mathrm{p}-1$, which can be decreased by $\mathrm{k}$ if a knot or a control point has multiplicity $\mathrm{k}+1$.

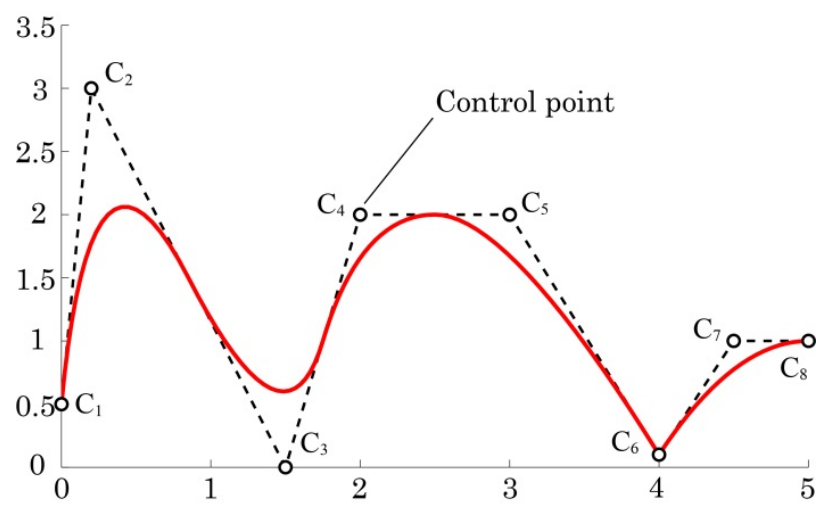

Figure 2. B-spline curve

\subsection{Refinements}

The h-, p- and k-refinement (Cottrell et al., 2009) schemes can be used in isogeometric analysis.

The h-refinement is a strategy that inserts knots without changing a curve geometrically or parametrically. This subdivision strategy changes the control points but maintains the order of basis functions. The p-refinement is a strategy that increases the polynomial order of basis functions without changing the geometry and parameterization of a curve. This strategy changes both the control points and the order of basis functions but knot span is not increased. The $\mathrm{h}$ - and $\mathrm{p}$ - refinement strategies are analogous to the classical $\mathrm{h}$ - and $\mathrm{p}$ - refinement strategies in finite element analysis. Finally, the k-refinement that is efficiency and robustness over the above instances is strategy that first elevates the order of original curve and only then inserts a unique knot values, unlike h-, p-refinement strategy. This strategy would enhance the continuity between the elements and have a higher regularity at new element interfaces than h-, p-refinement strategy. In this study, we define the element as the span between two distinct knot values.

\section{ISOGEOMETRIC FORMULATION}

In the absence of external load and damping effects, the dynamic equilibrium equation based on principle of virtual work can be written as

$$
\int_{0}^{L} E^{L} u_{, x} \delta u_{, x} d x=\int_{0}^{L} \delta u \rho A \ddot{d} d x
$$

where $\mathrm{E}$ is Young's modulus, $\mathrm{A}$ is the cross sectional area, $\mathrm{u}$ is the displacement, $\ddot{u}$ is the acceleration, $\rho$ is the density of material and the notation $\delta$ denotes that the terms are virtual.

The relevant derivation takes place in finite-dimensional subspace 
to turn the above virtual statement of the problem into a system of algebraic equations. In this study, the subspaces are defined by using the B-spline basis:

$$
\mathrm{u}=\sum_{\mathrm{a}=1}^{\mathrm{n}_{\mathrm{c}}} \mathrm{N}_{\mathrm{a}} \mathrm{u}_{\mathrm{a}}, \quad \ddot{\mathrm{u}}=\sum_{\mathrm{a}=1}^{\mathrm{n}_{\mathrm{c}}} \mathrm{N}_{\mathrm{a}} \ddot{\mathrm{u}}_{\mathrm{a}}
$$

where $n_{c}$ is the total number of the control point in the discretized domain and the virtual terms associated with the displacement and acceleration are

$$
\delta \mathrm{u}=\sum_{\mathrm{a}=1}^{\mathrm{n}_{\mathrm{c}}} \mathrm{N}_{\mathrm{a}} \delta \mathrm{u}_{\mathrm{a}}, \quad \delta \ddot{\mathrm{u}}=\sum_{\mathrm{a}=1}^{\mathrm{n}_{\mathrm{c}}} \mathrm{N}_{\mathrm{a}} \delta \ddot{\mathrm{u}}_{\mathrm{a}} .
$$

Substituting (5) and (6) into (4) yields

$$
\delta \mathbf{u}^{\mathrm{T}}[\mathbf{K u}-\mathbf{M} \ddot{\mathbf{u}}]=0 .
$$

Since the virtual displacement $\delta \mathrm{u}$ is arbitrary, the above equation may be written as

$$
\mathbf{K u}-\mathbf{M} \ddot{\mathbf{u}}=\mathbf{0}
$$

A general solution of (8) may be written

$$
\mathrm{u}=\phi_{k} \mathrm{e}^{i \omega_{k}}
$$

Substituting (9) into (8) yields

$$
\left[\mathbf{K}-\omega_{k}^{2} \mathbf{M}\right] \phi_{k}=0
$$

where $\phi_{k}$ is a set of displacement-type amplitude at the control points otherwise known as the model vector. $\omega_{\mathrm{k}}$ is the natural frequency associated with the $\mathrm{k}^{\text {th }}$ mode and $\mathrm{K}$ and $\mathrm{M}$ are global stiffness and mass matrices which contain contributions from element stiffness and mass matrices.

The structural stiffness and mass matrices in (10) can be explicitly written as

$$
\begin{array}{r}
\mathbf{K}=K_{a b}=E A \int_{0}^{L} \nabla_{x} N_{a, p} \nabla_{x} N_{b, p} d x \\
\mathbf{M}=M_{a b}=\int_{0}^{L} \mathrm{~N}_{a, p} \rho \mathrm{N}_{a, p} d x
\end{array}
$$

where $\mathrm{a}, \mathrm{b}$ is the number of control points used in the structural geometric model, $\nabla_{x}$ is the differential operator $\partial() / \partial x, \rho$ is the density of material, $\mathrm{N}_{\mathrm{a}, \mathrm{p}}$ is the basis function of the order $\mathrm{p}$ associated with the control point a.

\section{SOME REMARKS ON IMPLEMETATION}

To formulate the stiffness matrix in element level, let us use coordinate systems as shown in Figure 3.

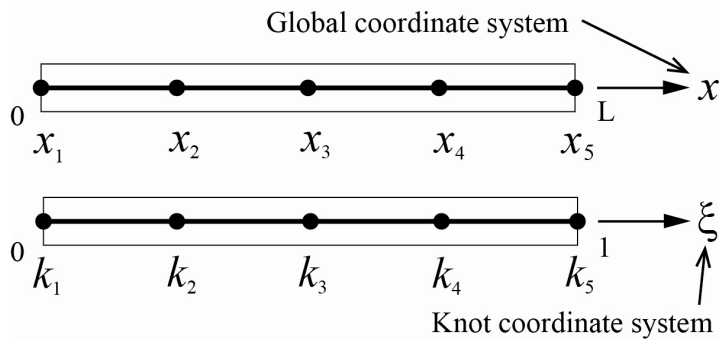

Figure 3. Coordinate systems

In the knot coordinate system, knot spans between not-repeating knots in the knot vectors become the integration ranges for the calculation of element stiffness matrices. For example, the stiffness and mass matrices for elastic bar, where the essential boundary conditions are not considered, can be written as

$$
\begin{gathered}
\mathbf{K}=\prod_{i=1}^{n_{\text {span }}}\left[K_{a b}\right]^{(i)}=\prod_{i=1}^{n_{\text {span }}} E A \int_{x_{\text {span }}}^{x_{i+1}} \nabla_{x} N_{a, p} \nabla_{x} N_{b, p} d x, \\
\mathbf{M}=\prod_{i=1}^{n_{\text {span }}}\left[M_{a b}\right]^{(i)}=\prod_{i=1}^{x_{i+1}} \int_{x_{i}} N_{a, p} \rho N_{b, p} d x .
\end{gathered}
$$

Let the span is assumed to be as an isogeometric element and then the above equation can be written in the knot coordinate system as follows

$$
\begin{gathered}
\prod_{e=1}^{n_{e l}}\left[\mathrm{~K}_{a b}\right]^{(e)}=\prod_{e=1}^{n_{e l}} E A \int_{k_{e}}^{k_{e+1}} \frac{1}{\mathrm{~J}} \nabla_{\xi} N_{a, p} \frac{1}{\mathrm{~J}} \nabla_{\xi} N_{b, p} \operatorname{det}(\mathrm{J}) d \xi, \\
\prod_{e=1}^{n_{e l}}\left[\mathrm{M}_{a b}\right]^{(e)}=\prod_{e=1}^{n_{e l}} \int_{k_{e}}^{k_{e+1}} \mathrm{~N}_{a, p} \rho \mathrm{N}_{b, p} \operatorname{det}(\mathrm{J}) d \xi .
\end{gathered}
$$

where $\mathrm{n}_{\mathrm{el}}\left(=\mathrm{n}_{\text {span }}\right)$ is the number of element (or knot span), $\left[\mathrm{k}_{\mathrm{e}} \mathrm{k}_{\mathrm{e}+1}\right]$ is knot interval for integration, J is Jacobian matrix between $\mathrm{x}$ and $\xi$, $a, b$ is the basis function number associated with the target span (or element) and $\mathrm{p}$ is the order of basis function.

At this point, it should be noted that the first-order basis functions $\left(\mathrm{N}_{\mathrm{b}, 1}\right)$ is exactly the same as linear shape function used in FE method and so the element stiffness matrix obtained from the first order of basis function is identical to the element stiffness matrix of 2-node bar FE element.

Thus, as an example, the element stiffness matrix is here described by using the basis functions of the quadratic order. This quadratic basis function is described in the Table 1 and used to evaluate the stiffness matrix along with the knot vector $\Xi=[0,0,0,1,1,1]$. Basis functions of Table 1 are illustrated in Figure 4.

As shown in Figure 4, the knot span between two distinct knot values is $[0,1]$. Namely, the number of isogeometric element is 
Table 1. Basis functions with $\Xi=[0,0,0,1,1,1]$

\begin{tabular}{cc}
\hline Basis function & Knot span $[0,1]$ \\
\hline$N_{1,2}(\xi)$ & $(1-\xi)^{2}$ \\
$N_{2,2}(\xi)$ & $2 \xi(1-\xi)$ \\
$N_{3,2}(\xi)$ & $\xi^{2}$ \\
\hline
\end{tabular}

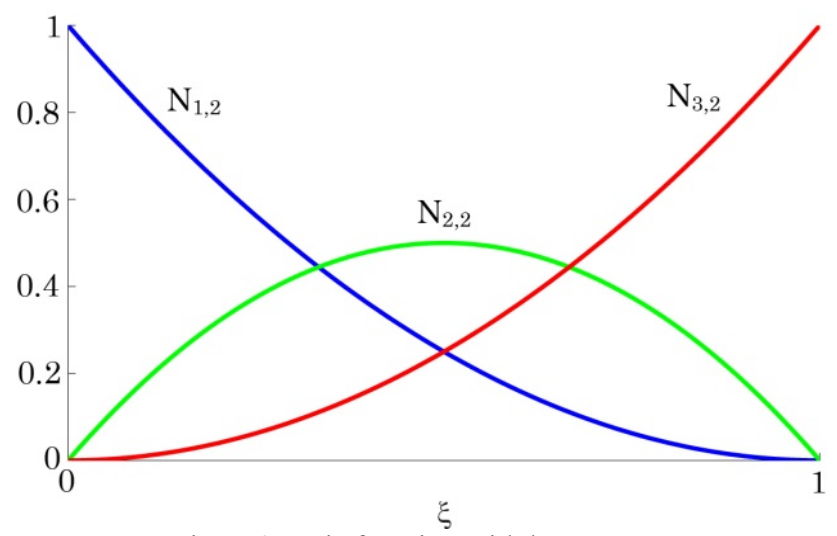

Figure 4. Basis function with knot vector $\Xi=[0,0,0,1,1,1]$

considered as one. To set up the B-spline curve, three control points are defined in the global coordinate system. Note that the control point is very important in the isogeometric analysis since the values of unknown displacements are directly associated with the control points.

For elastic bar element, each control point has one degree of freedom so that the size of element stiffness matrix using a quadratic-order basis function becomes $\mathrm{K}^{(\mathrm{e})} \in \mathrm{R}^{3 \times 3}$. If a $\operatorname{knot}(\xi=0.5)$ is inserted in the given knot vector $[0,0,0,1,1,1]$ via $\mathrm{h}$-refinement, the knot vector becomes $\Xi=[0,0,0,0.5,1,1,1]$. Then, the number of element is increased from one to two because the knot intervals between two distinct knot values become $[0,0.5]$ and $[0.5,1]$. In addition, the number of control points is also increased from three to four. Consequently, the new basis functions can be defined by (2). They are described in the Table 2 along with new knot vector $\Xi=[0,0,0,0.5,1,1,1]$.

Table 2. Basis functions with $\Xi=[0,0,0,0.5,1,1,1]$

\begin{tabular}{ccc}
\hline Basis function & $\begin{array}{c}\text { Knot span } \\
{[0,0.5]}\end{array}$ & $\begin{array}{c}\text { Knot span } \\
{[0.5,1]}\end{array}$ \\
\hline$N_{1,2}(\xi)$ & $(2 \xi-1)^{2}$ & 0 \\
$N_{2,2}(\xi)$ & $2 \xi(2-3 \xi)$ & $2(\xi-1)^{2}$ \\
$N_{3,2}(\xi)$ & $2 \xi^{2}$ & $(2$ \\
$N_{4,2}(\xi)$ & 0 & $-2 \xi)(3 \xi-1)$ \\
& 0 & $(2 \xi-1)^{2}$ \\
\hline
\end{tabular}

Basis functions of Table 2 are illustrated in Figure 5 .Now, the number of element (or span) is two in the knot vectors $\Xi=[0,0,0,0.5,1,1,1]$ so the assembly of element stiffness matrix is required to form the structural stiffness matrix.

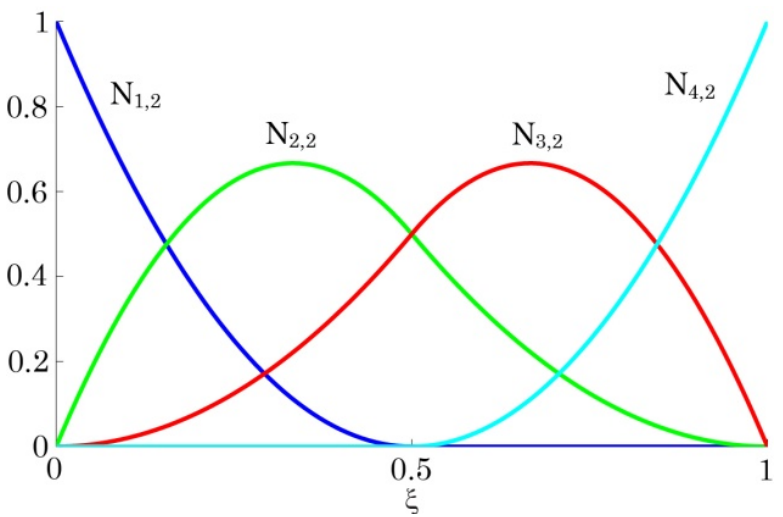

Figure 5. Basis functionwith knot vector $\Xi=[0,0,0,0.5,1,1,1]$

The stiffness matrices for knot intervals (or elements) are assembled by overlapping the control points in similar way of overlapping the nodes in the FE method (Hughes, 2000). In case of the knot vectors $\Xi=[0,0,0,0.5,1,1,1]$, the control points $\left[\mathrm{C}_{1}, \mathrm{C}_{2}, \mathrm{C}_{3}\right]$ is associated with the first knot interval (or the first element) [0 $0.5]$, and the control points $\left[\mathrm{C}_{2}, \mathrm{C}_{3}, \mathrm{C}_{4}\right]$ is associated with the second interval (or the second element) [0.5 1]. Therefore, the profile of structural stiffness matrix can be illustrated as shown in Figure 6.

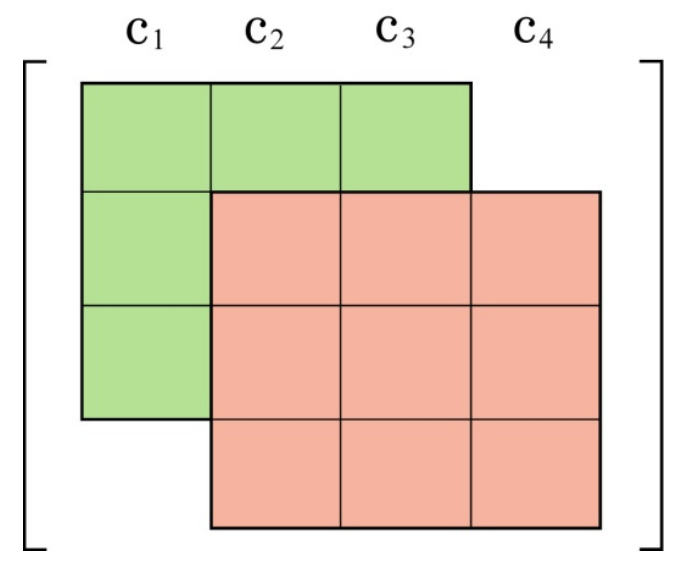

Figure 6. Structural stiffness matrix profile

\section{NUMERICAL TEST}

In this section, the efficiency and accuracy of the present isogeometric bar element is demonstrated by using numerical tests. For this purpose, some important aspects of isogeometric analysis are investigated under free vibration condition: (1) the convergence rate of p-refinement; (2) the convergence rate of h-refinement and (3) the convergence rate of k-refinement. The fixed-fixed bar and cantilever bar are employed in this test.

\subsection{Fixed-fixed bar}

The fixed-fixed bar is analyzed. The geometry of the bar is illustrated in Figure 7. The following material properties are used: $\mathrm{E}=10^{8}, \mathrm{~A}=1, \mathrm{~L}=2, \rho=1$. All units are assumed to be consistent. 


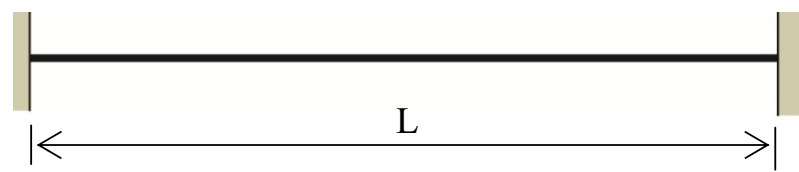

Figure 7. Geometry of fixed-fixed bar

The convergence rate of h-refinementis investigated for the first six frequencies. We begin with a single isogeometric element and then insert the knots to increase the number of element. Five orders of basis function ( $\mathrm{p}=1,2,3,4$ and 5 ) are employed and six isogeometric element meshes (1, 2, 4, 8, 16 and 32 elements) are used. Knot vectors used for h-refinement are summarized in Table 3. Note that we strictly use the open knot vector so that the first and last components of knot vector have $\mathrm{p}+1$ multiplicity.

Table 3. Knot vectors for h-refinement

\begin{tabular}{c|l}
\hline Elements $(\mathrm{n})$ & The components of knot vectors \\
\hline 1 & 0,1 \\
\hline 2 & $0,0.5,1$ \\
\hline 4 & $0,0.25,0.5,0.75,1$ \\
\hline 8 & $0,0.125,0.25,0.375,0.5,0.625,0.75,0.875,1$ \\
\hline \multirow{2}{*}{16} & $0,0.0625,0.125,0.1875,0.25,0.3125,0.375,0.4375$, \\
& $0.5,0.5625,0.625,0.6875,0.75,0.8125,0.875$, \\
\hline 32 & $0.9375,1$ \\
\hline
\end{tabular}

The convergence rates of the first three frequencies $\left(\omega_{1}, \omega_{2}, \omega_{3}\right)$ due to the h-refinement are illustrated in Figure 8. From Figure 8, we can see that linear order of basis function $(p=1)$ have the same convergence rate with linear bar FE. Here, it should be noted that the first order basis function is exactly same as the linear Lagrange interpolation function (Hughes, 2000). However, for higher orders of basis function such as $\mathrm{p}=3,4$ and 5 , the complete convergence

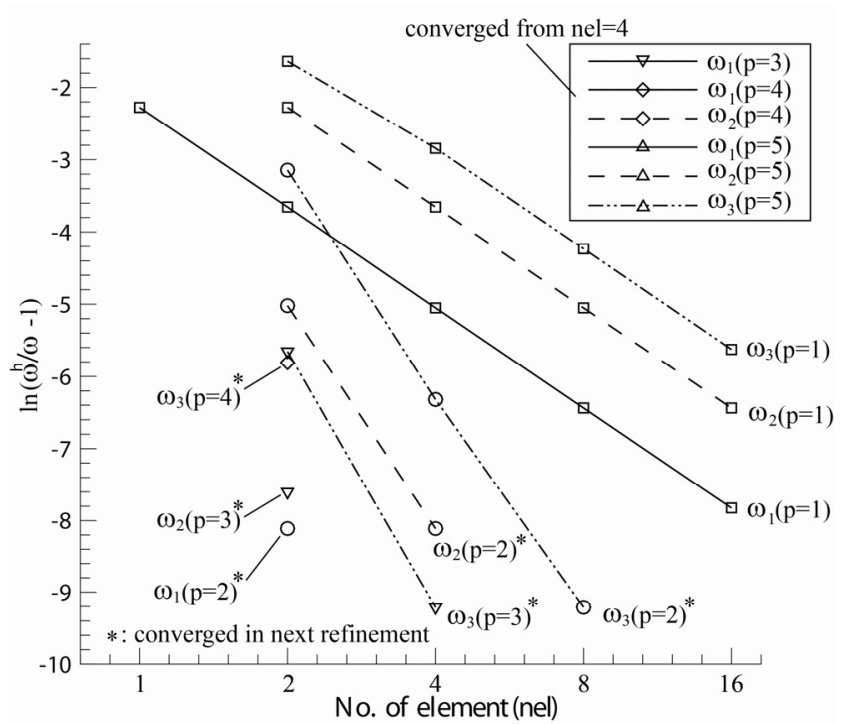

Figure 8. Convergence rates of the first three frequencies $\left(\omega_{1}, \omega_{2}, \omega_{3}\right)$ for the present isogeometric element due to the h-refinement

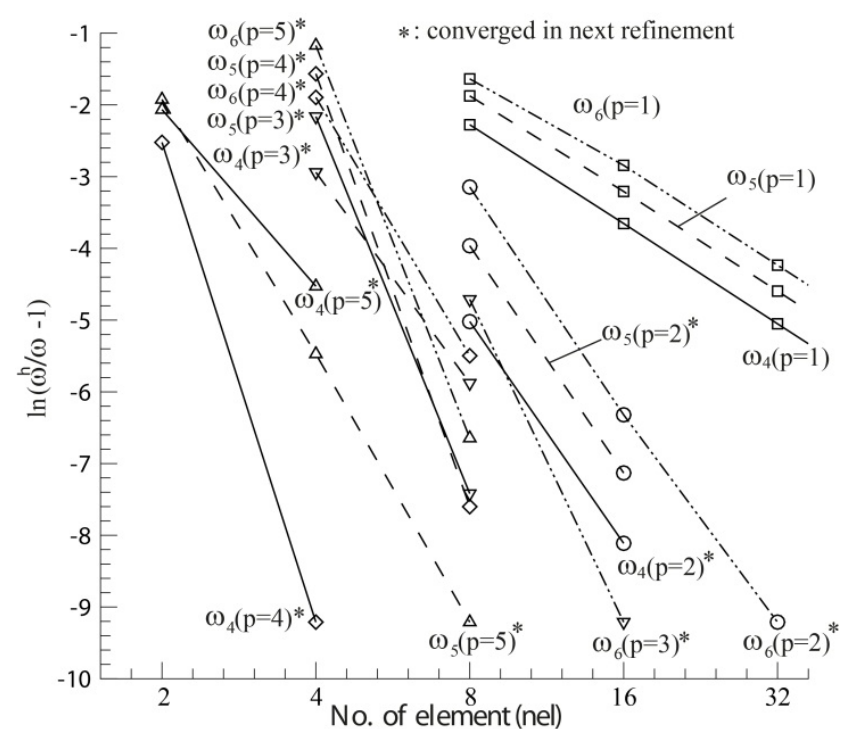

Figure 9. Convergence rates of the second three frequencies $\left(\omega_{4}, \omega_{5}, \omega_{6}\right)$ for the present isogeometric element due to the h-refinement

is achieved by using around eight isogeometric elements. In particular, if we use the orders $p=4$ or 5 , the frequencies $\left(\omega_{1}, \omega_{2}\right)$ are all converged by using only four isogeometric elements. The convergence rates of the second three frequencies $\left(\omega_{4}, \omega_{5}, \omega_{6}\right)$ due to the h-refinement are also calculated and illustrated in Figure 9.

As shown in Figure 9, the frequencies associated with higher modes $\left(4^{\text {th }}, 5^{\text {th }}\right.$ and $6^{\text {th }}$ modes $)$ require more elements than those of lower modes $\left(1^{\text {st }}, 2^{\text {nd }}, 3^{\text {rd }}\right.$ modes $)$ to achieve the convergence to the exact solutions. When we use the higher orders of basis function, all the frequencies seem to converge to the exact solution with less than sixteen isogeometric element. But with lower orders of basis function such as $p=1$ and 2 , we require more isogeometric elements for the convergence. However, we should remember that an appropriate number of control points are necessarily required to keep a good accuracy in the isogeometric solutions.

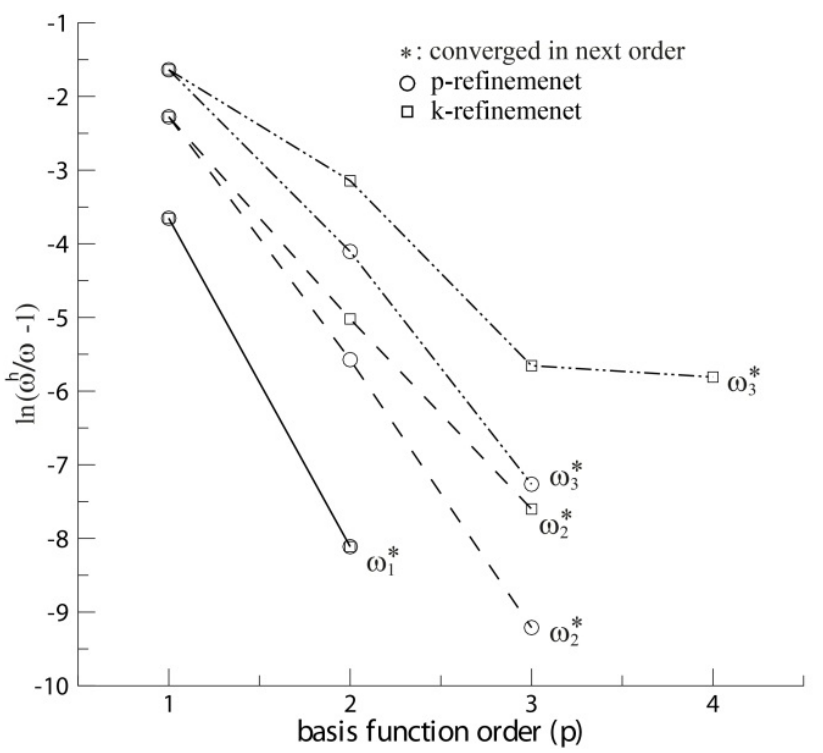

Figure 10. Convergence rates of the first three frequencies for the present isogeometric element due to the p- and k-refinement 
We then investigate the convergence rate of the present isogeometric element due to $\mathrm{p}$ - and k-refinement. For this purpose, we use four isogeometric elements for the analysis. The five different orders of basis function such as $\mathrm{p}=1,2,3,4$ and 5 are used in this test. We calculate the first three frequencies of the $\operatorname{bar}\left(\omega_{1}, \omega_{2}, \omega_{3}\right)$ and illustrated in Figure 10.

From numerical results, we can see that the increase of the order of basis function can speed up the convergence of the isogeometric solution for both $\mathrm{p}$ - and k-refinement. In this test, the convergence of fundamental frequency $\left(\omega_{1}\right)$ to exact solution is achieved at the order $p=3$. The convergences of higher frequencies $\left(\omega_{2}, \omega_{3}\right)$ are also achieved at the order $p=4$ except the third frequency $\omega_{3}$ with the $\mathrm{k}$-refinement which is converged at the order $\mathrm{p}=5$. Now, the frequency spectra are produced by the present isogeometric element and illustrated in Figure 11.

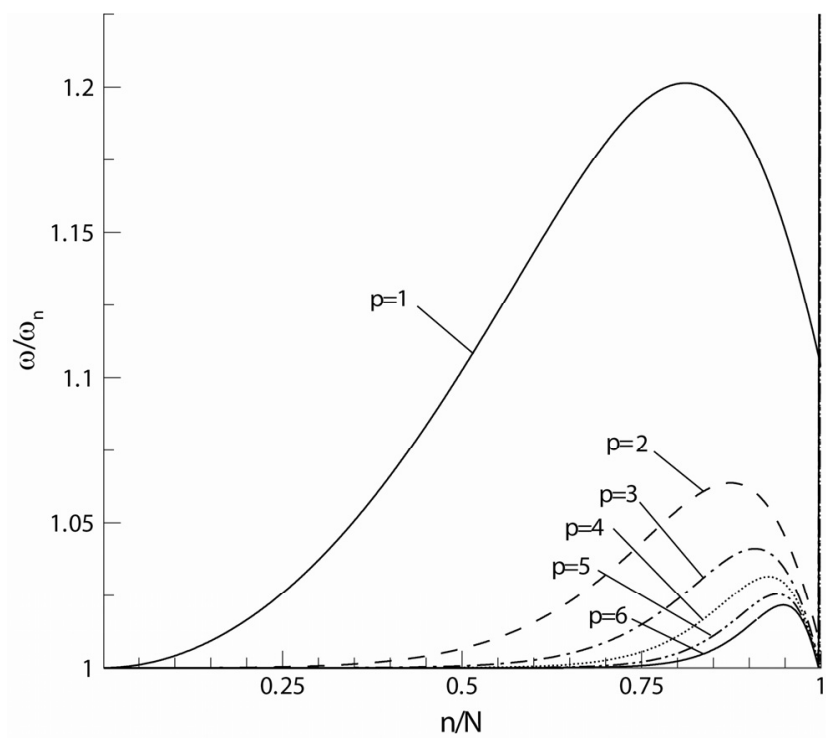

Figure 11. Frequency spectra for fixed-fixed bar

In the spectra, all the natural frequencies are normalized by the exact solutions. For this calculation, we use $\mathrm{N}=1024$ isogeometric elements with six different orders of basis function such as $\mathrm{p}=1$, $2,3,4,5$ and 6 . A very smooth shape of the frequency spectra is obtained.We found that the order of basis function can greatly effect on the accuracy of natural frequency values. In particular, the higher order of basis function can guarantee the accuracy of isogeometric solutions.

\subsection{Cantilever bar}

In this example, cantilever bar is analyzed with the same material properties used in Section 5.1. The geometry of cantilever bar is illustrated in Figure 12.

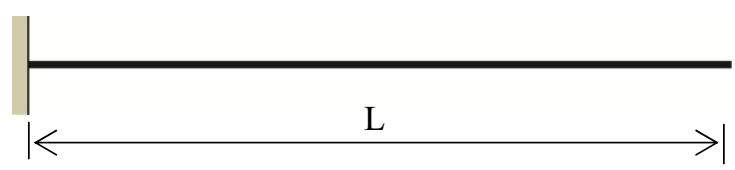

Figure 12. Geometry of cantilever
We here investigate the convergence of the present isogeometric element due to the h-, p- and k-refinements.

For h-refinement, we use the same knot vector described in Table 3. Numerical results are illustrated in Figures 13 and 14.

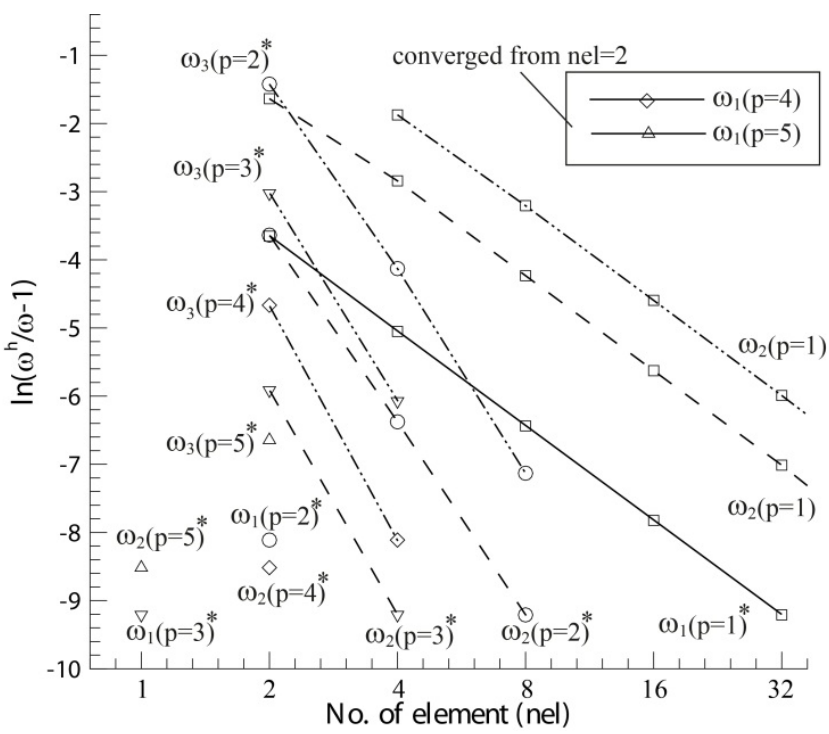

Figure 13. Convergence rates of the first three frequencies $\left(\omega_{1}, \omega_{2}, \omega_{3}\right)$ for the present isogeometric element due to the h-refinement

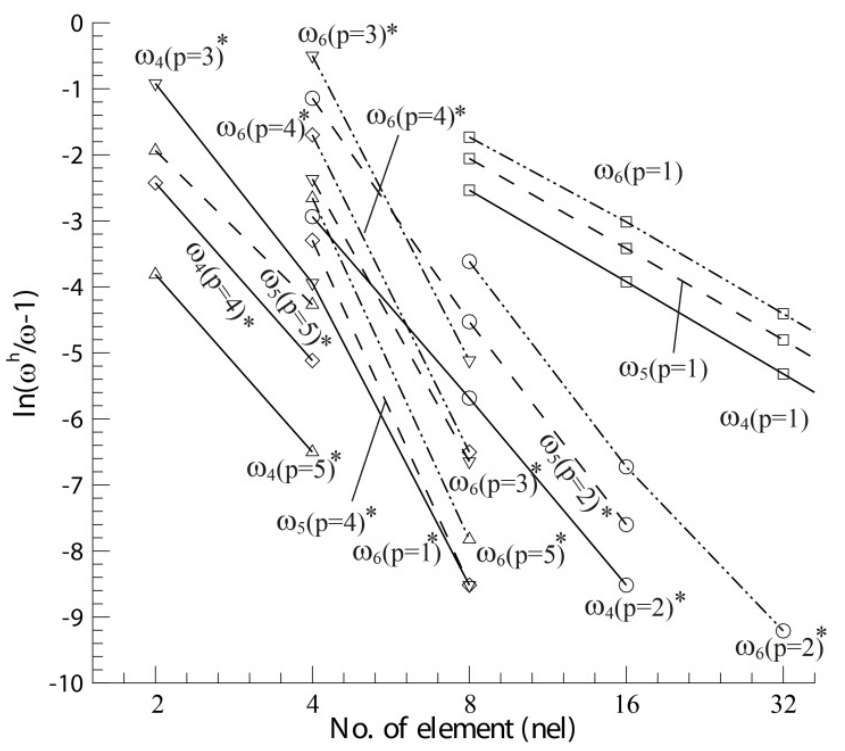

Figure 14. Convergence rates of the second three frequencies $\left(\omega_{4}, \omega_{5}, \omega_{6}\right)$ for the present isogeometric element due to the h-refinement

The convergence rate of the present element appears to be a similar to those of fixed-fixed bar. However, the fast convergence is mostly achieved compared to the fixed-fixed bar. In particular, if we use the higher orders of basis function such as $p=4,5$, the convergence is achieved with only less than eight elements for most frequencies. In particular, the fundamental frequency is converged with only four elements. The numerical results for first three frequencies are illustrated in Figure 13. 
For the second three frequencies $\left(\omega_{4}, \omega_{5}, \omega_{6}\right)$, the convergence rate is also similar to those of fixed-fixed bar. However, fast convergence rate is achieved compared to that of the fixed-fixed bar as illustrated in Figure 14. For further detailed investigation may require for higher frequencies of the bar.

We also investigate the convergence rate of the present isogeometric element due to p- and k- refinements. See Figure 15.

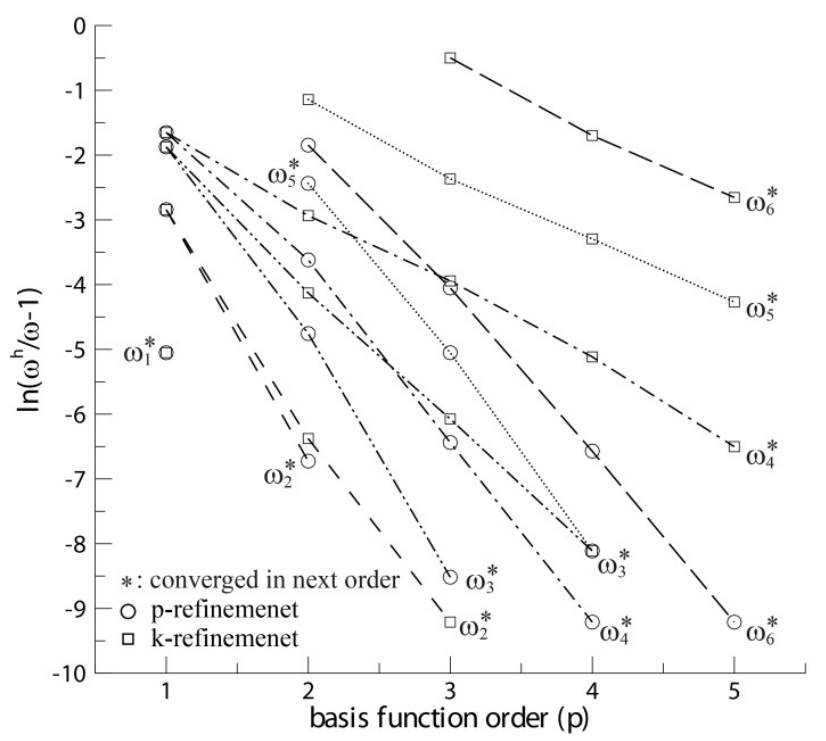

Figure 15. Convergence rates of the first three frequencies for the present isogeometric element due to the $\mathrm{p}$ - and $\mathrm{k}$-refinement
3. Higher order of basis function can lead to faster convergence for most frequencies.

4. Consistent mass matrix formed by using Gaussian quadrature rule can provide accurate isogeometric solution.

It is found to be that the present isogeometric element has a powerful and versatile capability to produce very accurate natural frequencies of bar structures and is applicable to most of bar structures. Finally, the present isogeometric solutions described in this paper are provided as future reference solutions.

\section{REFERENCES}

Cottrell, J.A., Bazilevs, Y. and Hughes, T.J.R. (2009) Isogeometric Analysis: Towards Integration of CAD and FEA.Wiley.

De Boor, C. (1978)A Practical Guide to Splines. Springer.

Hughes, T.J.R. (2000)The Finite Element Method: Linear Static and Dynamic Finite ElementAnalysis. Dover Publications.

Hughes,T.J.R. Cottrell, J. A. and Bazilevs, Y. (2005) Isogeometricanalysis: CAD, finiteelements, NURBS, exact geometry andmesh refinement.Comput. Methods Appl. Mech. Eng., vol. 194, no. 39-41, pp. 4135-4195.

Hughes, T.J.R. and Evans J.A. (2010) Isogeometric analysis.ICES Report 10-18, The Institute of Computational Engineering and Science, University of Texas Austin

Rogers,D. (2001) An Introduction to NURBS: With Historical Perspective.Academic Press, London, UK.

(Date of Submission : 2011.5.17)

For both refinements, very fast convergence rate is achieved in calculation of fundamental frequency $\left(\omega_{1}\right)$. It is also found to be that the higher order of basis function can also guarantee the fast convergence on most modes. Faster convergence rate is detected by using p-refinement than k-refinement when we use the same isogeometric element. However, it should be noted that the larger number of control point is generally required for $\mathrm{p}$-refinement compared to k-refinement when the same number of isogeometric element is used. However, the convergence is achieved at the same order for both p-refinement and k-refinement. The frequency spectra are also produced for this example and it is found to be almost identical to that of cantilever bar.

\section{CONCULSIONS}

An isogeometric bar element is developed and used to analyze bar structures under free vibration. The description of isogeometric bar formulation for free vibration analysis and its implementation aspect is consistently provided here. Numerical tests are carried out to demonstrate the efficiency and accuracy of present isogeometric bar element. Here, some specific conclusions are drawn from numerical tests:

1. The present isogeometric bar element does not have any restriction on the choice of order of basis function for free vibration analysis.

2. No particular effort is required to perform the h-,pand k-refinements during isogeometric analysis. 